\title{
Intégrité des données scientifiques : transparence des données recueillies au cours des essais cliniques, accès aux données
}

\author{
Nicholas Moore ${ }^{1}$, Yves Juillet ${ }^{2}$, Pierre-Henri Bertoye ${ }^{3}$ et les participants de la table ronde $n^{\circ} 4$ de Giens XXII \\ 1 Département de Pharmacologie, Université de Bordeaux, Bordeaux, France \\ 2 Les Entreprises du Médicament, Paris, France \\ 3 Agence Française de Sécurité Sanitaire des Produits de Santé, Afssaps, Saint Denis, France
}

\begin{abstract}
Mots clés :
essais cliniques fraude; malversations ; prévention; gestion

Résumé - L'intégrité des données recueillies dans les essais cliniques et leur utilisation sont un élément fondamental de la démarche scientifique, et de la confiance que l'on peut avoir dans cette démarche. Les exemples de fraudes sont légion, et régulièrement répétés. L'objectif de cette table ronde était de faire le point sur la définition de la fraude, sur sa reconnaissance et sa prévention, en particulier dans le système institutionnel. La fraude implique une intention de tromper et va du camouflage, de l'incompétence à l'invention de toutes pièces d'études, de patients ou de données. Sa fréquence est difficile à évaluer, mais semblerait atteindre au moins $1 \%$ des études ou publications. Cette fraude peut impliquer l'éthique (avis du CPP [comité de protection des personnes] a posteriori, non-respect des demandes du CPP, défaut de consentement) ou toutes les étapes de la réalisation à l'interprétation des essais ou études. L'identification des fraudes est rendue difficile par le risque pris par l'«avertisseur» (whistleblower), qu'il faut protéger. La recherche de la fraude est implicite dans les bonnes pratiques cliniques que devraient appliquer tous les promoteurs industriels mais qui est moins souvent mise en application par les promoteurs institutionnels. Il conviendrait donc de prévoir des recommandations pour la mise en place de procédures de détection des fraudes en particulier dans les études institutionnelles. Diverses méthodes statistiques peuvent être utilisées pour détecter les invraisemblances suggestives de fraude. Une fois la fraude identifiée, sa gestion n'est en général pas prévue ou anticipée. Ici encore des procédures ou recommandations devraient être proposées.
\end{abstract}

«Un jour pluvieux de novembre 2006, Eric Poehlmann se tenait devant un juge fédéral du Vermont, pour écouter sa condamnation à 5 ans de prison pour fraude. L'histoire a commencé quelques années auparavant, quand un de ses techniciens/étudiants a remarqué que des données qu'il avait analysées quelques temps avant avaient changé et que les résultats initialement négatifs étaient devenus positifs. Il a essayé de comprendre pourquoi ces données avaient changé et a confronté son patron qui l'a menacé de le virer. Il en a parlé à un collègue qui lui a recommandé de bien documenter ses suspicions s'il allait plus loin. Il a documenté toutes ses allégations, puis est allé devant l'administration de son université. L'enquête a duré cinq ans et a mis à jours des fraudes répétées et systématiques, mettant à bas toute la recherche sur les effets de l'âge et de la ménopause sur la masse grasse, et une dizaine de publications dans les meilleurs journaux.

\footnotetext{
* Pour la liste des participants, voir en fin d'article.
}

Eric Poehlmann avait reçu plusieurs dizaines de millions de dollars pour soutenir cette recherche, et divers prix pour les résultats. Il est apparu que la fraude avait été perpétrée pour corriger des résultats qui ne confirmaient pas l'hypothèse originale dont le chercheur était convaincu, et qui paraissait biologiquement plausible. Exceptionnellement, l'avertisseur n'a pas perdu son emploi, si ce n'est que l'équipe de recherche qui l'employait a disparu. Eric Poehlmann est en prison » (extrait de «An unwelcome discovery », Jeneen Interlandi, New York Times, 22 October 2006).

Dans ce cas comme dans beaucoup d'autres similaires (mais rarement de cette ampleur), c'est un assistant ou un collègue qui pose des questions sur la validité de résultats, et qui amène la découverte de la fraude. Au-delà bien sûr de l'opprobre qui s'abat sur le fraudeur, et la destruction fréquente de la carrière éventuelle de l'avertisseur souvent initialement dénoncé, ${ }^{[1]}$ cette fraude est très préjudiciable pour d'autres chercheurs, qu'ils soient dans 
la même équipe ou qu'ils s'appuient sur ces résultats frauduleux. Ceci peut également entraîner des conséquences à distance, parce que des projets de recherche sont financés, fondés sur ces résultats frauduleux, et ces finances ne sont donc plus disponibles pour d'autres études. Plus grave, d'autres pistes de recherche, peut-être plus difficiles à poursuivre risquent d'être à tort délaissées, que ceci soit dans le domaine clinique ou pré-clinique. Enfin la fraude en discréditant la recherche et ses résultats entachent de doutes la recherche authentique et honnête. ${ }^{[2]}$

La fraude dans la recherche est un phénomène aussi ancien que la recherche elle-même, et qui a fait l'objet de nombreux ouvrages. ${ }^{[3-5]}$ Cette fraude ou ces malversations touchent aussi bien l'industrie pharmaceutique ${ }^{[6]}$ que la recherche académique. $^{[7,8]}$ Tous les jours de nouveaux cas de fraude sont rapportés dans la grande presse, et chaque nouveau cas entraîne des conséquences néfastes pour tous les acteurs impliqués, mais également pour la recherche. ${ }^{[9]}$

La fraude, et les moyens de l'identifier de la traiter, de la prévenir sont l'objet de cette table ronde, justifiée par ces exemples récents de fraude scientifique.

\section{Définition et champ, contexte}

La fraude est un acte de mauvaise foi accompli en contrevenant à la loi ou aux règlements et nuisant au droit d'autrui : il s'agit donc d'un acte intentionnel, ce qui exclut a priori l'incompétence, qui ne l'est pas.

L'importance du problème est difficile à évaluer faute de données précises. Les fraudes visibles ou rendues publiques représenteraient environ $1 \%$ des essais ou publications. ${ }^{[10]}$ En cas de suspicion de fraude investiguée, environ $50 \%$ des suspicions sont confirmées. ${ }^{[11]}$ Un point ressort constamment, celui de la prise de risque par l'avertisseur, ce qui nous amène à une première recommandation : protéger l'avertisseur, par le développement des points suivants : (i) le constat initial doit être factuel et documenté ; (ii) un cadre réglementaire rendant obligatoire la déclaration en cas de suspicion devrait être établi.

La table ronde n'a pas fait de particularisme sur les conflits d'intérêts et n'a pas travaillé sur les biais volontaires de protocole établis en vue d'orienter les résultats. ${ }^{[12]}$

\section{Fraude sur l'Ethique}

Il s'agit d'avis de Comité de Protection (CPP) faux ou établis a posteriori, de consentements truqués, de signatures imitées, de modifications demandées par le CPP non respectées mais présentées comme satisfaites...

Deux grandes classes de fraudes ont été identifiées. (a) Les avis a posteriori ou les faux avis de CPP en particulier pour des études qui ne relèvent pas d'un avis de CPP (études non interventionnelles), en raison d'un désir de publication et d'une exigence d'avis de « comité d'éthique ». Bien qu'il s'agisse d'une fraude stricto sensu, il n'y a pas de défaut de consentement ni de risque non évalué pour les patients participant à la recherche.

La recommandation est d'obtenir des rédacteurs de journaux l'acceptation d'un avis CCTIRS (Comité Consultatif sur le Traitement de l'Information en Matière de Recherche dans le Domaine de la Santé, également appelé pré-CNIL), Commission nationale informatique et libertés (CNIL) ou Conseil national de l'ordre des médecins (CNOM) comme équivalent à un avis de comité d'éthique, pour les études ne relevant pas en France d'un avis de CPP.

(b) Des cas où effectivement les recommandations du CPP n’ont pas été mises en œuvre ou les patients n'ont pas donné leur consentement : il y a une faute directe concernant l'éthique, le consentement ou la protection des personnes.

Que faut-il faire?

- Informer les investigateurs et le promoteur en cas d'étude multicentrique?

- Informer les autres CPP si le ou les CPP de la région de l'investigateur fraudeur n'est en particulier pas celui de l'investigateur coordonnateur : il est en effet possible que le même investigateur soit impliqué dans d'autres études?

- Que faire de ces données obtenues de façon non éthique? Ces résultats, lorsqu'ils sont scientifiquement valables, sont-ils à écarter d'emblée ou pas?

Deux cas de figures ont été identifiés, aboutissant à une recommandation :

- si on peut circonscrire clairement les données non éthiques, faire l'analyse avec, puis sans les données non éthiques et comparer les résultats obtenus ;

- si l'on NE peut PAS isoler ces données, soit ne pas utiliser du tout l'étude, soit l'utiliser (avec accès contrôlé ?) en mentionnant le caractère non éthique du recueil, en particulier lorsqu'il s'agit de résultats qui ne pourront pas être reproduits, et qui ont des conséquences importantes en santé publique (SIDA par exemple);

- dans tous les cas, engager des poursuites judiciaires envers les responsables de l'essai.

\section{Fraudes dans les données et les résultats des essais cliniques}

Ces fraudes peuvent être divisées en fraudes concernant le recueil des données et fraudes concernant leur analyse (en particulier analyse statistique). 
L'identification et la gestion des fraudes dans les données relèvent en général du monitorage habituel des essais cliniques, et en principe les données frauduleuses identifiées sont retirées des analyses de l'étude.

Une seconde zone de fraude est dans l'analyse statistique de l'étude, et en particulier la possibilité d'une analyse statistique orientée en fonction des résultats. Il peut également arriver que des résultats intermédiaires donnent des résultats différents des résultats finaux : on peut donc être amené à se demander pourquoi et si ces différences sont licites. Il convient donc de pouvoir reproduire les différentes étapes de l'analyse qui ont fourni les différents résultats.

Recommandation :

- d'un plan d'analyse formalisé, daté, traçable avec les dates des amendements ;

- d'une base de données gelées traçable et archivée au moment d'une production de résultats pour en permettre la reconstitution si nécessaire.

\section{Détection de la fraude}

Les acteurs d'un essai sont multiples et les contrôles sont à mettre en œuvre tout au long de la chaîne de réalisation, de la promotion à la publication : le promoteur/prestataire de services, le contrôle de qualité par les moniteurs, l'audit, les autorités, dont l'inspection et l'évaluation, les collaborateurs de l'investigateur ou les statisticiens, les revues, les référés, les co-auteurs, les lecteurs avertis. ${ }^{[13]}$ Chacun de ces acteurs peut être amené, comme cela a été prouvé dans le passé, à identifier une suspicion de fraude.

Si la recherche de fraude ou d'irrégularité est partie intégrante de l'activité de promoteurs industriels, et de l'évaluation par les autorités réglementaires qui revoient les essais soumis pour l'enregistrement des médicaments, il en est souvent bien autrement dans les travaux à financement public, et dont le promoteur est l'hôpital public ou certain Etablissements publics scientifiques ou techniques (EPST). Le promoteur, notamment institutionnel, n'a souvent pas vraiment pris conscience du problème ou n'a pas les moyens de l'aborder (figure 1), et ce d'autant plus que l'investigateur est un leader d'opinion. Ces travaux peuvent donner lieu à publication dans des revues de très haut niveau, et entraîner une perturbation importante des domaines scientifiques concernés.

Classiquement la détection des fraudes revient surtout au monitorage et à l'audit.

Le détection des fraudes passe également par l'analyse statistique, en particulier dans les essais multicentriques, des différences entre centres par exemple. ${ }^{[13,14]}$ Dans certains cas (cahiers d'observation électroniques [case report form $=\mathrm{CRF}$, i pour internet et e pour électronique], iCRF ou eCRF), les modifications successives des saisies peuvent être indicatrices : il convient donc d'en conserver la trace (notion de piste d'audit).

La recherche académique ou institutionnelle est souvent ignorante des méthodes et techniques de détection des fraudes.

Recommandations :

- adéquation des moyens et méthodes au risque (de fraude) de l'essai ;

- monitorage ciblé ;

- indicateurs de cohérence intersites (essai multicentrique);

- audit des cahiers d'observation électroniques ;

- mise au point d'un guide pour la détection des fraudes.

\section{Traitement du signal : investigation, démonstration de la fraude}

Une fois que la fraude est suspectée, il faut l'investiguer pour en démontrer la réalité : une suspicion non démontrée reste une tache potentielle. Il faut que l'enquête ne mette pas en danger inutilement la réputation d'un investigateur suspecté à tort, sans pour autant protéger excessivement un investigateur coupable. L'expérience de ces procédures n'est pas développée. Il n'est également pas toujours facile pour une institution d'investiguer ses propres membres, et le risque de conflits d'intérêts est grand. Il serait utile qu'il y ait au minimum des procédures standardisées décrivant la gestion d'un signal de fraude suspectée ou avérée, et permettant de gérer de la manière la plus objective possible les cas suspects garantissant pendant l'enquête la présomption d'innocence. Au mieux pourrait être instituée une instance d'investigation indépendante conduisant ces enquêtes à la demande de l'institution.

Recommandation :

- mise en place d'un Bureau National de l'Intégrité de la Recherche, qui serait une structure d'assistance aux institutions de recherche à la fois dans le domaine de la prévention et dans la conduite à tenir vis-à-vis d'un signal.

\section{Gestion de la fraude}

Quoi faire du fraudeur une fois la fraude fortement suspectée ou prouvée ? Faut-il faire intervenir l'Université, l'Hôpital, l'Afssaps, la Justice, les Ordres professionnels, les CPPs, l'industriel? Ou toutes ces instances et dans quel ordre ?

Face à une fraude avérée, le niveau d'intervention, le besoin d'une intervention purement professionnelle ou au contraire judiciaire n'est pas clair pour l'institution qui oscille souvent entre le 
a) lorsque le CHU est promoteur, a-t-il mis en place un monitorage systématique incluant une évaluation du risque de fraude ou de malversation?

Non $18 / 23$

monitorage systématique $5 / 23$

b) est-ce que vos URC et statisticiens ont reçu une formation spéciale à la détection de fraude?

non $22 / 23$

oui $1 / 23$

mais cela fait partie du métier d'ARC

c) y a-t-il une formation spécifique des investigateurs sur la fraude dans les essais cliniques?

Non 22/23

mais souvent rappel dispositions légales lors de la mise en place du projet

d) Combien de cas de fraude suspectée/confirmée avez-vous identifié depuis 5 ans, parmi les projets dont vous êtes promoteur

\section{9: 0 fraudes ; 4: 1-4 fraudes; 1: 20\% déviations mineures}

e) en cas de fraude avérée, quelle est votre attitude?

- ne rien faire?

- mettre en garde la personne contre une récidive?

- la « blacklister » (interdit de PHRC, par exemple) ?

- informer la CME/la commission de discipline/l'université/le Conseil de l'ordre ?

- porter plainte en justice?

- dépend de la fraude

\section{1 : non applicable}

Commentaire : les DRC de CHU estiment le plus souvent ne pas avoir les moyens d'agir.

Fig. 1. Résultats d'une enquête auprès des DRC de $\mathrm{CHU}$, concernant les attitudes des $\mathrm{CHU}$ sur la fraude. Dans l'encadré, les questions posées; en gras, les réponses. 23 sur les $31 \mathrm{DRC}$ contactées ont répondu.

URC : unité de recherche clinique; ARC : assistant de recherche clinique; PHRC : programme hospitalier de recherche clinique; CME : commission médicale d'établissement; DRC : Direction de la recherche clinique; CHU : Centre hospitalier universitaire.

désir de prévention par l'exemple et la crainte du scandale. Certains industriels auront peut-être plutôt tendance à se contenter de ne plus faire appel à l'investigateur. D'une manière générale, comme dit plus haut, il serait préférable pour tous qu'une décision claire de justice sur la réalité ou non de la fraude soit prise. Il faudrait un mode d'emploi, des instructions claires et partagées sur la gestion de ce risque.

Recommandation :

- procédures communes de prise en charge du fraudeur avéré, rendues publiques a priori;

- ceci rejoint également le point 1 relatif au cadre réglementaire rendant obligatoire la déclaration administrative en cas de suspicion de fraude.

\section{Prévention de la fraude}

\section{1. Le dossier médical personnel}

Le Dossier Médical Personnel (DMP) et la carte Vitale sont importants pour enregistrer et documenter la participation du pa- tient à un essai (et donc, outre les aspects relatifs à sa sécurité, en vérifier l'existence et les modalités de sa participation). Le suivi des actes médicaux et dates permet un suivi objectif de l'étude et de la réalité des visites, consultations et actes à la fois pour détecter les différences éventuelles avec les cahiers d'observation ou les dates déclarées des visites d'essai. De plus cela permet une identification de cette activité pour le médecin traitant ou tout autre professionnel de santé ou pour éviter l'inclusion par erreur du même patient dans plusieurs essais simultanés. Cette utilisation ne pourrait bien évidemment être envisagée qu'avec l'accord explicite du malade.

Recommandation :

- inclure les données des essais dans le DMP.

\subsection{Formation des acteurs des essais}

Une part de la fraude vient d'un manque de formation des investigateurs ou des autres acteurs de la recherche qui à la suite d'une erreur méthodologique ou de réalisation pourraient 
être tentés de rattraper ou de masquer l'erreur par recours à la fraude. ${ }^{[15,16]}$ Tous les efforts doivent être faits pour améliorer la qualité du travail par la formation. ${ }^{[17]}$ La formation permet également d'augmenter la sensibilité à la fraude et donc sa détection par les co-investigateurs, ou par la lecture critique de la littérature.

Cette formation existe, mais est-elle utilisée?

Un Corpus de formation minimal commun devrait être défini, incluant une formation spécifique, partie intégrante de la formation initiale, outre l'analyse critique d'articles dans l'Examen National Classant, et des formations complémentaires adaptées : (i) formation spécialisée : mastère, formation assistants recherche clinique (FARC), formation des investigateurs aux essais cliniques (FIEC); (ii) formation continue : EPP validants (évaluation des pratiques professionnelles).

Cette formation devrait concerner toutes les personnes impliquées dans les essais cliniques, et non seulement l'investigateur principal, et chacun à son propre niveau, tant pour les groupes d'investigation (Investigateurs, assistants recherche clinique [ARC], techniciens d'études cliniques [TEC], Direction recherche clinique [DRC], pharmacies hospitalières, laboratoires, ...) que pour les structures de promotion. Typiquement ces formations pourraient être proposées, coordonnées ou harmonisées dans le sein de l'inter-région par la Délégation Interrégionale à la Recherche Clinique (DIRC).

Recommandation :

- mise en place d'un corpus de formation minimal commun pour les acteurs présents, dans le cadre notamment de la formation initiale mais aussi continue;

- formation à destination de tous les acteurs d'essais, pas seulement les investigateurs principaux;

- l'étude de cas simulés peut être un outil précieux.

\section{Aspects juridiques}

D'une manière générale, les ressources juridiques dont on dispose en France pour poursuive les auteurs de ces fraudes reposent sur le droit pénal ou le droit administratif (Code Pénal/Code de la santé publique, dont le Code de déontologie médicale). Le droit de la santé publique est spécifique à cette situation de recherche (absence de consentement par exemple) alors que le droit pénal est non spécifique : les délits ou fautes concernés peuvent être par exemple faux et usage de faux, escroquerie pour l'acteur principal, recel et complicité pour ceux qui seraient informés mais n'agiraient pas.

Les sanctions sont l'emprisonnement, des amendes, l'interdiction d'exercice, avec des tarifs variables qui vont par exemple de 5 ans de prison et $375000 €$ d'amende pour escroquerie ou de 3 ans de prison, $45000 €$ d'amende pour recherche sans consentement. La même faute peut être poursuivie à divers titres, et une analyse juridique et d'opportunité soigneuse peut permettre de définir la stratégie la plus appropriée.

Quelle que soit la voie juridique utilisée, la communication sur la fraude et sur la condamnation est fondamentale $:^{[18]}$ un médecin interdit d'exercice pourrait annoncer sa décision d'aller «faire le tour du monde » sans en donner la raison et donc masquer sa condamnation.

Recommandation :

- publicité des jugements ;

- publicisation sur sites officiels des jugements et peines encourues.

\section{La fraude dans les publications}

Finalement les principales causes fondamentales de la fraude sont l'appât du gain (fraude dans les études à promotion industrielle), la recherche de la gloire (recherche académique), ces deux motivations n'étant pas exclusives. La recherche académique aboutira le plus souvent à une publication, et c'est souvent le désir d'améliorer la probabilité d'acceptation d'un article dans une grande revue qui sera à l'origine de la fraude. La fraude pourra donc avoir, de par la publication de résultats falsifiés, des conséquences majeures sur tout l'environnement de la recherche qui s'appuierait sur ces résultats faux pour développer des actions ou stratégies ultérieures en particulier dans le domaine de la santé publique.

La publication a donc une double importance dans la fraude en recherche : un point de détection de la fraude, et une victime de l'activité frauduleuse. Il faudra en particulier veiller à ce qu'une fois que la fraude est détectée, tous les travaux du même investigateur soient soigneusement examinés, et que surtout le lecteur et le monde de la recherche soient clairement avertis de la fraude ou du risque de fraude.

Une enquête rapide analogue à celle des hôpitaux a été conduite auprès de certains journaux, soit généralistes de grande diffusion à équipe éditoriale professionnelle, soit plus spécialisés à équipe bénévole, sur leur organisation quant à la détection de la fraude. Aucune différence sensible n'a été identifiée entre ces deux types de revues, malgré des déclarations publiques parfois fracassantes (figure 2).

Il n'y a donc que peu ou pas de processus systématique de détection de fraude, qui concerne 1-3\% des publications (au moins). ${ }^{[11]}$ Ce sont surtout des plagiats et duplication. Il n'y a pas de rétractation systématique, c'est-à-dire de mentions postérieures justifiant le fait que l'étude publiée n'était pas la bonne. 


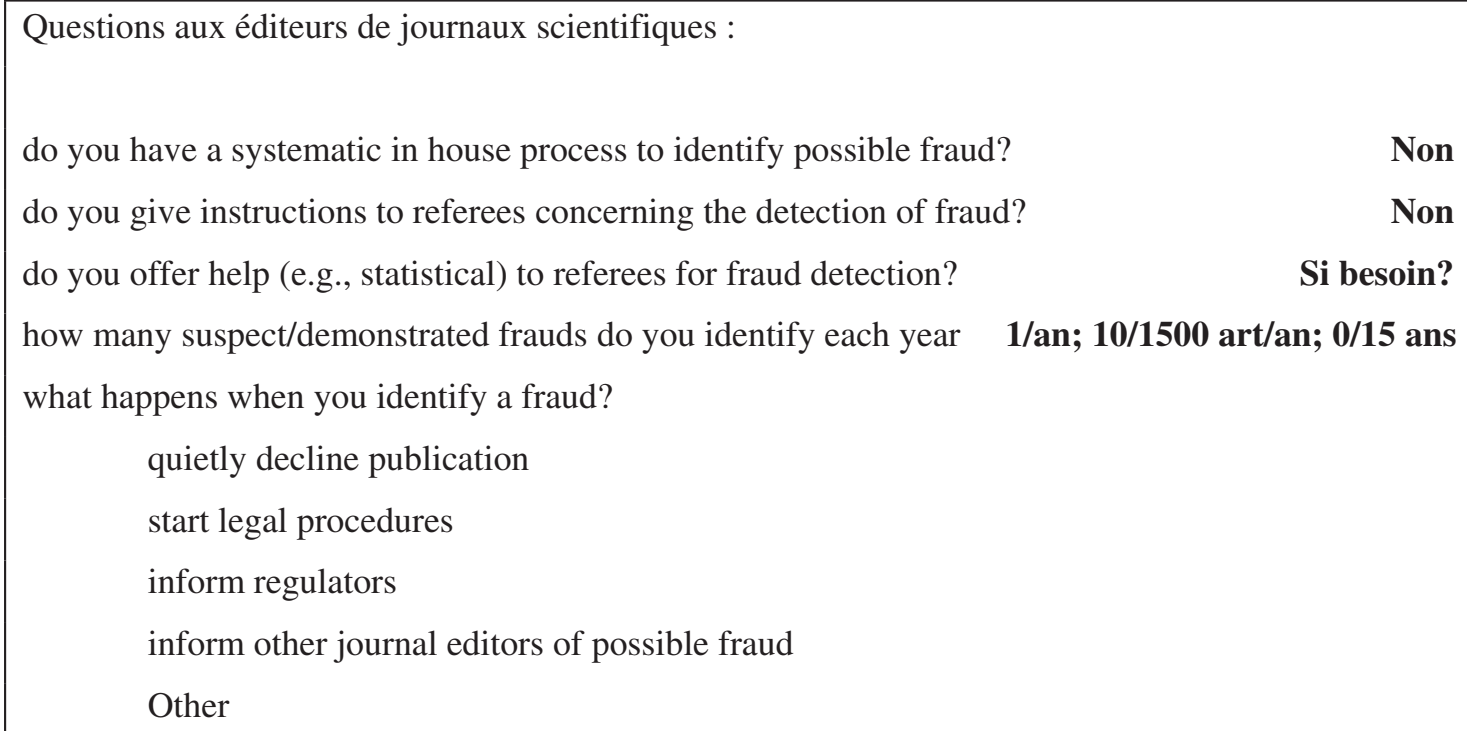

Fig. 2. Réponses à un questionnaire posé à des rédacteurs de revues scientifiques. Une dizaine ont répondu, dont des revues généralistes de très haut niveau, et des revues plus spécialisées. art = articles.

\section{Recommandation :}

- mise en place de procédures d'identification des fraudes concernant les référés ou les rédacteurs : accès au protocole, données brutes, ... si suspicion (gestion confidentielle);

- une fois la fraude avérée, rétractation des articles et/ou identification claire dans les bases du caractère frauduleux de la publication.

Un guide de bonnes pratiques d'éthique de publication, décrivant ces procédures est librement accessible. ${ }^{[19]}$

\section{Mise en place de procédures d'identification de fraudes par la mise en place de registres}

La mise en place de registres d'essais, avec l'objectif de la transparence des informations, la visibilité de l'adéquation entre les méthodes et les résultats permet de limiter en particulier les fraudes liées au masquage des résultats ou à leur interprétation orientée.
Certains de ces registres existent déjà : Clinicaltrials, ${ }^{[20]}$ portail IFPMA. ${ }^{[21]}$

D'autres sont en cours de réalisation : registres Eudrapharm européen, registre national en France.

Recommandation :

- procédure facilitée de diffusion des informations entre registres, facilitant la recherche multi-registres.

\section{Conclusion}

En conclusion, la fraude est un problème certainement très sous-estimé, et insuffisamment traité, par manque de prévention, de vigilance, et d'exploitation adaptée. Il peut toucher tous les types d'essais à tous les points de la conception à la réalisation à l'analyse ou l'interprétation. Le point qui est constamment revenu est le souhait de disposer de procédures publiques aidant en particulier les institutionnels, mais également les promoteurs privés pour la détection et surtout la gestion de la fraude suspectée ou sa prévention. Ce sera également la recommandation commune globale de la table ronde. 


\section{Participants}

Christophe Baduel (GlaxoSmithKline, Marly le Roi), Corinne Bernaud (AstraZeneca, Rueil Malmaison), Geneviève Chêne (INSERM U 593, Bordeaux), Dominique Doucet (Afssaps, Saint Denis), Thérèse Dupin-Spriet (Faculté Pharmacie, Lille), Valérie Foltzer (Coherences Pharma), Jean-Marie Goehrs (Merck Sharp \& Dohme Chibret, Paris), Danielle Golinelli (Direction Générale de la Santé, Paris), Sylvie Hansel (Hôpital Lapeyronie, Montpellier), Jean-Marc Husson (Eudipharm, Paris), Pascale Jolliet (Institut de Biologie, Nantes), Rémi LeCoent (GlaxoSmithKline, Marly le Roi), Joël Moret-Bailly (Sainte Foy les Lyon), François Pelen (Pfizer, Paris), Yannick Plétan (Pfizer, Paris), Catherine Rey-Quino (Afssaps, Saint Denis), Jean-Charles Reynier (AP-HM, CHRU, Marseille), Tabassome Simon (Faculté de Médecine Saint Antoine, Paris), Anne-Priscille Vlasto (Leem, Paris), Beat Widler (Hoffmann Laroche AG, Basel, Suisse), Faiez Zannad (CIC, Hôpital Jeanne d'Arc, Dommartin les Toul)

\section{Références}

1. Rhodes R, Strain JJ. Whistleblowing in academic medicine. J Med Ethics 2004; 30(1): 35-9

2. Kansu E, Ruacan S. Research ethics and scientific misconduct in biomedical research. Acta Neurochir Suppl 2002; 83: 11-5

3. de Pracontal M. L'imposture scientifique en dix leçons. Paris: Seuil; 2005

4. Lock S, Wells F. Fraud and misconduct in medical research. London: BMJ books; 2002

5. Broad W, Wade N. La souris truquée. Enquête sur la fraude scientifique. Paris: Seuil; 1987

6. Mathews AW. Fraud, errors taint a key study of widely used Sanofi drug; despite some faked results, FDA approves antibiotic; one doctor's cocaine use; company defends safety. Wall St J (East Ed) 2006: A1, A12
7. Couzin J, Schirber M. Scientific misconduct. Fraud upends oral cancer field, casting doubt on prevention trial. Science 2006; 311(5760): 448-9

8. Mathews AW, Wonacott P. Playing detective: at medical journal, editor finds truth hard to track down; termite-eaten data plague Dr. Smith's 12-year probe of an Indian researcher. Wall St J (East Ed) 2005: A1, A2

9. Al-Marzouki S, Roberts I, Marshall T, et al. The effect of scientific misconduct on the results of clinical trials: a Delphi survey. Contemp Clin Trials 2005; 26(3): 331-7

10. Ranstam J, Buyse M, George SL, et al. Fraud in medical research: an international survey of biostatisticians. ISCB Subcommittee on Fraud. Control Clin Trials 2000; 21(5): 415-27

11. Gardner W, Lidz CW, Hartwig KC. Authors' reports about research integrity problems in clinical trials. Contemp Clin Trials 2005; 26(2): 244-51

12. Safer DJ. Design and reporting modifications in industry-sponsored comparative psychopharmacology trials. J Nerv Ment Dis 2002; 190(9): 583-92

13. Buyse M, George SL, Evans S, et al. The role of biostatistics in the prevention, detection and treatment of fraud in clinical trials. Stat Med 1999; 18(24): 3435-51

14. Al-Marzouki S, Evans S, Marshall T, et al. Are these data real? Statistical methods for the detection of data fabrication in clinical trials. BMJ 2005; 331(7511): 267-70

15. Bostanci A, Vogel G. Research misconduct. German inquiry finds flaws, not fraud. Science 2002; 298(5598): 1531-3

16. DeMets DL. Distinctions between fraud, bias, errors, misunderstanding, and incompetence. Control Clin Trials 1997; 18(6): 637-50; discussion 661-6

17. Nylenna M, Simonsen S. Scientific misconduct: a new approach to prevention. Lancet 2006; 367(9526): 1882-4

18. Dyer O. GP struck off for fraud in drug trials. BMJ 1996; 312 (7034): 798 [letter]

19. http://blackwellpublishing.com/publicationethics/

20. http://www.clinicaltrials.gov

21. http://www.ifpma.org/clinicaltrials

Correspondance et offprints : Nicholas Moore, Département de Pharmacologie, Université de Bordeaux, 33076 Bordeaux Cedex, France.

E-mail : nicholas.moore@pharmaco.u-bordeaux2.fr 